\title{
The Role of Seeing Blood in Non-Suicidal Self-|njurry
}

\author{
Catherine R. Glenn
}

Stony Brook University

\section{E. David Klonsky \\ University of British Columbia}

Non-suicidal self-injury (NSSI) is a growing clinical problem, especially among adolescents and young adults. Anecdotal accounts, clinical reports, and popular media sources suggest that observing the blood resulting from NSSI often plays an important role in the behavior's reinforcement. However, research to date has not systematically assessed the role of blood in NSSI. The current study examined this phenomenon in 64 young adults from a college population with histories of non-suicidal skin-cutting. Approximately half the participants reported it was important to see blood during NSSI. These individuals reported spending five minutes or less looking at the blood after each instance of NSSI, and that seeing blood served several functions including "to relieve tension" and "makes me feel calm." In addition, wanting to see blood was associated with greater lifetime frequency of skin-cutting and greater endorsement of intrapersonal functions for NSSI (e.g., affect regulation, self-punishment). Finally, participants who reported wanting to see blood were more likely to endorse symptoms of bulimia nervosa and borderline personality disorder. Theoretical and clinical implications are discussed. (c) 2010 Wiley Periodicals, Inc. J Clin Psychol 66: 466-473, 2010.

Keywords: self-injurious behavior; deliberate self-harm; non-suicidal self-injury; skin-cutting; self-mutilation; self-damaging behaviors; blood

Non-suicidal self-injury (NSSI; e.g., skin-cutting, burning) refers to the direct, deliberate injuring of body tissue without suicidal intent. Although NSSI is common in psychiatric samples, recent studies have also found high rates in adolescent and young adult populations (Ross \& Heath, 2002; Whitlock et al., 2006), and these rates appear to be increasing over time (Briere \& Gil, 1998). High, and potentially increasing, rates of NSSI are alarming because of NSSI's association with severe

Correspondence concerning this article should be addressed to: E. David Klonsky, University of British Columbia, Department of Psychology, 2136 West Mall, Vancouver, B.C. V6T 1Z4 Canada; e-mail: edklonsky@gmail.com 
psychopathology, including anxiety, depression, borderline personality disorder, and suicidality (Andover, Pepper, Ryabchenko, Orrico, \& Gibb, 2005).

Although research has begun to illuminate factors that cause NSSI and maintain the behavior over time (e.g., most common NSSI motivation is affect regulation; Klonsky, 2007), there is still much about the behavior's nature and functions that remains poorly understood. One salient but poorly understood aspect of NSSI is the role of blood. Evidence from a variety of non-empirical sources (e.g., popular media and clinical reports) suggests that seeing blood during NSSI contributes to the behavior's reinforcement. For example, many popular songs include lyrics about blood during NSSI, such as, "So when I feel the need, I think it's time to bleed. I'm gonna cut myself and watch the blood hit the ground" (Scherr \& Walker, 2003), and "Yeah you bleed just to know you're alive" (Rzeznik, 1998). Beyond popular media, a number of books on NSSI contain anecdotal accounts regarding the role of blood in self-injury. In Bodies Under Siege (1987), Dr. Armando Favazza states that one way NSSI produces relief is by releasing "bad blood" from dysfunctional relationships (p. 273). In Strong's (1998) A Bright Red Scream, a male [Lukas] describes the cleansing function of "blood-letting" (i.e., releasing blood during NSSI) as follows: "I cut secondarily for the pain, primarily for the blood... Watching the blood pour out makes me feel clean, purified" (p.11).

The role of seeing blood during NSSI has further appeared in a number of clinical reports. For example, following a series of interviews with self-injurers, Himber (1994) discusses the role of blood in NSSI as indicating a "good" cut; that is, seeing the blood appears to signify that the NSSI was performed correctly. Solomon and Farrano (1996) also reported on the role of blood in a series of NSSI case studies. In one, a 17-year-old adolescent reported that "seeing the blood... makes me feel calmer" (p. 113). To date, Favazza and Conterio (1989) provide the best empirical data on the topic. Although not the main focus of the study, $47 \%$ of a female sample of self-injurers reported that it was comforting to see their blood and $25 \%$ reported that they liked to taste their blood. Taken together, these reports suggest that the desire to see blood during NSSI is relatively common, and that seeing blood may be an "active ingredient" that helps NSSI achieve the desired effect, specifically, the reduction of unwanted and unpleasant affect states (e.g., to feel calmer or to feel alive). In addition, blood may also help to indicate that the cutting was deep enough or performed "correctly."

Although anecdotal evidence and clinical reports about the role of blood in NSSI are ample and salient, there has been little systematic research on the role of blood in NSSI. The purpose of the current study was to examine the phenomenon of seeing blood in NSSI, including its prevalence, functions, and clinical correlates. Based on the evidence presented above, we hypothesize that seeing blood in NSSI is a common practice that serves a variety of functions from relieving unpleasant emotions to indicating that the NSSI was performed properly.

\section{Method}

\section{Participants and Procedure}

Sixty-four young adults who engaged in non-suicidal skin-cutting were recruited from a mass screening administered to college students in lower-level psychology courses. Of the 1,100 students screened using the Inventory of Statements About Self-Injury (ISAS; see the Measures section), 216 (19.4\%) endorsed having used one 
method of NSSI at least once. Approximately half of these participants $(n=125)$ expressed general interest in a psychology study and $82(65.6 \%)$ agreed to participate when they were informed that the study was about NSSI. Of the final sample of 82 self-injurers, data from the 64 self-injurers who had engaged in skin-cutting were analyzed for the purposes of the present study.

The university's institutional review board approved the project and participant consent was obtained prior to the assessment. The 64 skin-cutters who qualified for inclusion completed the study in one lab visit. First, a brief structured interview was utilized to confirm presence of NSSI (see the Measures section). Next, participants completed the self-report questionnaires in paper-and-pencil format (i.e., ISAS, Patient Health Questionnaire [PHQ], McLean Screening Instrument for Borderline Personality Disorder [MSI-BPD]). Finally, the remainder of a brief structured interview for NSSI was administered by a masters-level graduate student.

\section{Measures}

ISAS (Klonsky \& Glenn, 2009; Klonsky \& Olino, 2008). The ISAS measures the frequency and functions of NSSI. Recent research found the ISAS to be a reliable and valid measure of NSSI frequency and functions in a large sample of young adults (Klonsky \& Glenn, 2009; Klonsky \& Olino, 2008). The first section of the ISAS assesses the lifetime frequency of 12 different NSSI behaviors performed "intentionally (i.e., on purpose) and without suicidal intent," including banging/ hitting, biting, burning, carving, cutting, interfering with wound healing, pinching, pulling hair, rubbing skin against rough surfaces, severe scratching, sticking self with needles, and swallowing dangerous substances. This section of the ISAS was used as the screening measure to recruit self-injurers.

The second section of the ISAS measures the functions of non-suicidal self-injury. The ISAS assesses 13 functions of NSSI that have been proposed in the empirical and theoretical mental health literature (Klonsky, 2007). The 13 functions of NSSI fall into two superordinate factors: (a) intrapersonal functions (i.e., affect regulation, anti-dissociation, anti-suicide, marking distress, and self-punishment) and (b) interpersonal functions (i.e., autonomy, interpersonal boundaries, interpersonal influence, peer bonding, revenge, self-care, sensation seeking, and toughness). Each function is assessed with 3 items that are rated on a scale from 0 (not at all relevant) to 2 (very relevant) to the experience of NSSI. Therefore, each of the 13 functional subscale scores ranges from $0-6$. The two superordinate scales (i.e., intrapersonal and interpersonal) are derived by summing the subscales that belong to each superordinate scale (see above) and then dividing by the number of subscales in order to obtain a mean score.

Brief Structured Interview for Non-Suicidal Self-Injury. A brief structured clinical interview for NSSI was designed for this study to confirm participant engagement in NSSI and to assess the role of blood in NSSI. The first section of the interview confirmed the history of NSSI. The second section of the interview assessed four variables regarding the role of blood during NSSI. The variables assessed were as follows: (a) whether it is important for an individual to see blood during a skin-cutting episode (yes or no); (b) (for those answering yes to item (a) how long they look at the blood (less than 1 minute, 1-5 minutes, 5-10 minutes, or more than 10 minutes); (c) the role seeing blood serves (i.e., beyond the overall function of NSSI; the following 6 functions were rated on a yes/no scale: relieves 
tension, makes me feel calm, makes me feel real, shows me that self-injury is real, helps me focus, and did it right/deep enough/time to stop); and finally, (d) how often an individual has fainted after seeing their blood during NSSI (never, sometimes, or always).

PHQ (Spitzer, Kroenke, \& Williams, 1999). The PHQ, an 83-item self-report questionnaire that assesses the Diagnostic and Statistical Manual of Mental Disorders (DSM-IV) symptoms associated with four types of psychological problems - anxiety, depression, eating, and substance/alcohol abuse - was used to measure Axis I clinical symptoms. The PHQ has demonstrated excellent convergence with independent practitioner ratings (.85) and good to excellent sensitivity (.75) and specificity (.90) in diagnosing DSM-IV Axis I disorders (Spitzer et al., 1999).

MSI-BPD (Zanarini et al., 2003). Borderline personality disorder (BPD) symptoms were assessed using the MSI-BPD, a 10-item self-report measure of BPD features. Compared with a validated structured interview, both sensitivity and specificity of the MSI-BPD were above .90 in a sample of young adults (Zanarini et al., 2003). A cut-off score of 7 or higher on the MSI-BPD yields the best sensitivity (.81) and specificity (.85) for a BPD diagnosis (Zanarini et al., 2003).

\section{Results}

The average age of participants was 19.08 (standard deviation $[S D]=1.90$ ) and the majority $(82.8 \%)$ were female. Approximately half of the sample $(51.6 \%)$ was Caucasian, followed by Asian (18.7\%), Hispanic (17.2\%), African American (3.1\%), and "other" or mixed ethnicities $(9.1 \%)$. Nearly half $(51.6 \%)$ of participants reported that it was important to see blood during NSSI (i.e., "Blood Important" group). (There were no significant differences in age, gender, or ethnicity between the "Blood Important" and "Blood Not Important" groups.) Of these participants, $42.4 \%$ reported looking at the blood for $1-5$ minutes, $33.3 \%$ for less than 1 minute, and $24.3 \%$ for more than 5 minutes. Only 1 participant ever fainted when seeing blood during NSSI. Most self-injurers $(84.8 \%)$ reported that seeing blood served multiple functions (mean $[M]=3.2, S D=1.4$, range $1-6$ ). The most strongly endorsed functions for seeing blood were relieves tension $(84.8 \%)$ and makes me feel calm (72.7\%). Other functions include makes me feel real (51.5\%), shows me that NSSI is real (42.4\%), helps me focus (33.3\%), and did it right/deep enough $(15.2 \%)$.

Next, the "Blood Important" and "Blood Not Important" groups were compared on characteristics relevant to the course and severity of NSSI (see Table 1). There were no differences between the two groups in the age of onset of NSSI $(t[62]=0.76$, $p=.45)$, total number of NSSI methods used $(t[62]=0.50, p=.62)$, or recency of NSSI (i.e., the last time they engaged in NSSI; $t[62]=0.80, p=.43$ ). The two groups were then compared on the frequency of cutting. The NSSI cutting data were converted to ranks because the distribution of skin-cutting contained a number of outliers. The "Blood Important" group engaged in significantly more cutting compared to the "Blood Not Important" group $(t[62]=4.23, p<.001)$. Specifically, the "Blood Important" group had cut themselves a median of 30 times compared with 4 times for the "Blood Not Important" group. Finally, we compared the functions reported for NSSI among those who did and did not report wanting to see blood. The "Blood Important" group endorsed significantly more intrapersonal 
Table 1

Means and Standard Deviations of NSSI and Clinical Measures for the "Blood Important" and "Blood Not Important" Self-Injuring Groups

\begin{tabular}{|c|c|c|c|}
\hline Variable $^{\mathrm{a}}$ & $\begin{array}{l}\text { All skin-cutting } \\
\text { self-injurers } \\
(n=64)\end{array}$ & $\begin{array}{l}\text { Blood } \\
\text { important } \\
(n=33)\end{array}$ & $\begin{array}{l}\text { Blood not } \\
\text { important } \\
(n=31)\end{array}$ \\
\hline \multicolumn{4}{|c|}{ Non-suicidal self-injury: (the ISAS and brief structured interview for NSSI) } \\
\hline Age of onset: Mean $(S D)$ & $13.13(2.91)$ & $13.39(2.93)$ & $12.84(2.92)$ \\
\hline $\begin{array}{l}\text { No. of NSSI methods } \\
\text { used: mean }(S D)\end{array}$ & $4.72(2.15)$ & $4.85(2.37)$ & $4.58(1.91)$ \\
\hline $\begin{array}{l}\text { Last time engaged in } \\
\text { NSSI: (in months) } \\
\text { mean ( } S D)\end{array}$ & $14.39(16.34)$ & $12.81(15.09)$ & $16.12(17.71)$ \\
\hline $\begin{array}{l}\text { Frequency of cutting }{ }^{\mathrm{b}}: \\
\text { median (range) }\end{array}$ & $15(1-1,000)$ & $30(2-350)$ & $4(1-1,000)^{* * *}$ \\
\hline $\begin{array}{l}\text { Intrapersonal/automatic } \\
\text { functions of NSSI: } \\
\text { mean }(S D)\end{array}$ & $2.80(1.15)$ & $3.35(1.03)$ & $2.22(0.98)^{* * *}$ \\
\hline $\begin{array}{l}\text { Interpersonal/social } \\
\text { functions of NSSI: } \\
\text { mean }(S D)\end{array}$ & $0.95(0.89)$ & $1.08(1.11)$ & $0.80(0.55)$ \\
\hline \multicolumn{4}{|c|}{ Axis I psychopathology ${ }^{\mathrm{c}}$ : (no. of participants who met full DSM-IV symptoms of disorder on PHQ) } \\
\hline Major depressive disorder & 12 & 8 & 4 \\
\hline $\begin{array}{l}\text { Generalized anxiety } \\
\text { disorder }\end{array}$ & 10 & 6 & 4 \\
\hline Bulimia nervosa & 5 & 5 & $0^{*}$ \\
\hline Binge eating disorder & 4 & 1 & 3 \\
\hline Alcohol abuse & 22 & 11 & 11 \\
\hline \multicolumn{4}{|c|}{ Axis II borderline personality disorder features: (items endorsed on the MSI-BPD) } \\
\hline $\begin{array}{l}\text { Total number of items: } \\
\quad \text { mean }(S D)\end{array}$ & $6.34(2.35)$ & $7.09(2.10)$ & $5.55(2.36)^{* *}$ \\
\hline $\begin{array}{l}\text { No. of participants who } \\
\text { endorsed } \geq 7 \text { items } \\
\quad \text { (i.e., BPD threshold) }^{\mathrm{c}}\end{array}$ & 37 & 25 & $12^{* *}$ \\
\hline
\end{tabular}

Note. ISAS = Inventory of Statements About Self-Injury; NSSI = Non-Suicidal Self-Injury; SD = standard deviation; PHQ = Patient Health Questionnaire; MSI-BPD = McLean Screening Instrument for Borderline Personality Disorder; DSM = Diagnostic and Statistical Manual of Mental Disorders.

aStatistical differences between the "Blood Important" and "Blood Not Important" self-injuring groups are indicated with ${ }^{*} p<.05,{ }^{* *} p<.01,{ }^{* * *} p<.001$.

${ }^{\mathrm{b}}$ Statistical tests used a rank-ordered cutting variable because of outliers; however, for purposes of the table, we report the median and range of the non-transformed cutting variable because these figures are more practically meaningful.

${ }^{\mathrm{c} C a t e g o r i c a l ~ g r o u p ~ d i f f e r e n c e s ~ w e r e ~ e x a m i n e d ~ u s i n g ~ a ~ P e a r s o n ~ c h i-s q u a r e ~ t e s t . ~}$

functions of NSSI (e.g., affect regulation) than the "Blood Not Important" group $(t[62]=4.49, p<.001)$. There was no difference in the endorsement of interpersonal functions of NSSI $(t[62]=1.27, p=.21)$.

Means and standard deviations of clinical variables are presented in Table 1. Although more participants in the "Blood Important" group endorsed symptoms of major depressive disorder and generalized anxiety disorder than in the "Blood Not Important" group, these differences did not reach statistical significance $(p=.25$ and $p=.56$, respectively). However, more members of the "Blood Important" group endorsed symptoms of bulimia nervosa than the "Blood Not Important" group, $\chi^{2}(1, N=64)=5.10, p<.05$. The "Blood Important" group also endorsed 
significantly more items on the MSI-BPD screening instrument for BPD, $t(62)=2.77, p<.01$, and had significantly more participants $(75.8 \%)$ who met the optimum cutoff for determining the presence of a BPD diagnosis (i.e., 7 or more items on the MSI-BPD) than the "Blood Not Important" group (38.7\%), $\chi^{2}(1, N=64)=9.00, p<.01$.

\section{Discussion}

This study examined the role of seeing blood in non-suicidal self-injury. In particular, we investigated the proportion of skin-cutters who reported that it was important to see blood during NSSI, the functions served by seeing blood, and the clinical characteristics that distinguish skin-cutters who find it important to see blood from those who do not. Findings suggest that wanting to see blood during non-suicidal skin-cutting is relatively common. Approximately half of participants reported that it was important to see blood during NSSI. There were no demographic differences between self-injurers who felt it was important to see blood and those who did not. Participants reported that seeing blood during non-suicidal skin-cutting served a number of functions, particularly to relieve tension and to calm down.

Notably, self-injurers who reported that it was important to see blood during NSSI were distinguished by certain clinical features. In regard to their self-injury, those who felt it was important to see blood were characterized by a higher frequency of skin-cutting and greater endorsement of intrapersonal functions for their NSSI (e.g., affect regulation). In addition, these self-injurers were more likely to endorse DSM-IV criteria for bulimia nervosa and borderline personality disorder. Overall, these results suggest that self-injurers who report it is important to see blood are a more clinically severe group of skin-cutters. Therefore, a desire to see blood during NSSI may represent a marker for increased psychopathology, a more persistent course of NSSI, and consideration of more aggressive treatment strategies.

Although findings from this study provide some insight into the role of blood in NSSI, an important question remains unanswered: What is the mechanism by which seeing blood during NSSI results in feelings of relief and/or calm (an effect reported by the majority of this self-injuring sample)? One potential mechanism is that the perception of blood leads to certain physiological changes, such as heart rate deceleration, that in turn lead to feelings of calm and relief. For example, previous studies have found that images and films involving blood (e.g., mutilation images or films of surgical procedures) initially produce a rapid deceleration in heart rate (Bradley, Codispoti, Cuthbert, \& Lang, 2001). Insofar as images of blood produce this physiological change, it stands to reason that seeing one's own blood during NSSI may initially lead to heart rate deceleration.

Another possible explanation for the effects of seeing blood during NSSI is parasympathetic rebound (i.e., strong parasympathetic activity following a sympathetic response to threat or danger). For example, studies of blood phobics suggest that seeing blood can induce a sympathetic response that is quickly followed by overcompensatory parasympathetic rebound (Friedman, 2007). From this perspective, seeing one's own blood may lead to an increase in sympathetic activity, which, in the absence of imminent threat, is quickly followed by a strong parasympathetic response. This parasympathetic response suppresses the effects of the sympathetic system (e.g., increased heart-rate) and associated emotions (e.g., anger, fear, panic) and promotes sustained attention and the regulation of emotions 
(i.e., producing a state of relaxation and calm; cf. Bradley \& Lang, 2007). Future studies should explore the mechanism by which seeing blood during NSSI produces relief; for example, studies could examine proxies for seeing one's own blood (e.g., red marker on skin) or actual blood (e.g., finger prick) in relation to measures of parasympathetic activity and subjective affect.

This study was the first to systematically examine the role of seeing blood during NSSI in a sample of self-injurers. Limitations of this study suggest areas for future research. In particular, future studies should replicate findings in younger and clinical samples using validated diagnostic interviews in addition to self-report measures.

\section{References}

Andover, M.S., Pepper, C.M., Ryabchenko, K.A., Orrico, E.G., \& Gibb. B.E. (2005). Selfmutilation and symptoms of depression, anxiety, and borderline personality disorder. Suicide and Life-Threatening Behavior, 35, 581-591.

Bradley, M.M., Codispoti, M., Cuthbert, B.N., \& Lang, P.J. (2001). Emotion and motivation I: Defensive and appetitive reactions in picture processing. Emotion, 1, 276-298.

Bradley, M.M., \& Lang, P.J. (2007). Emotion and motivation. In J.T. Cacioppo, L.G. Tassinary, \& G.G. Bernston (Eds.), Handbook of psychophysiology (pp. 581-607). New York: Cambridge University Press.

Briere, J., \& Gil, E. (1998). Self-mutilation in clinical and general population samples: Prevalence, correlates, and functions. American Journal of Orthopsychiatry, 68, 609-620.

Favazza, A.R. (1987). Bodies Under Seige: Self-mutilation and Body Modification in Culture and Psychiatry. Baltimore: Johns Hopkins University Press.

Favazza, A.R., \& Conterio, K. (1989). Female habitual self-mutilators. Acta Psychiatrica Scandinavica, 79, 282-289.

Friedman, B.H. (2007). An automatic flexibility-neurovisceral integration model of anxiety and cardiac vagal tone. Biological Psychology, 74, 185-199.

Himber, J. (1994). Blood rituals: Self-cutting in female psychiatric inpatients. Psychotherapy, 31, 620-631.

Klonsky, E.D. (2007). The functions of deliberate self-injury: a review of the evidence. Clinical Psychology Review, 27, 226-239.

Klonsky, E.D., \& Glenn, C.R. (2009). Assessing the functions of non-suicidal self-injury: Psychometric properties of the Inventory of Statements About Self-injury (ISAS). Journal of Psychopathology and Behavioral Assessment, 31, 215-219.

Klonsky, E.D., \& Olino, T.M. (2008). Identifying clinically distinct subgroups of self-injurers among young adults: A latent class analysis. Journal of Consulting and Clinical Psychology, 76, 22-27.

Ross, S., \& Heath, N. (2002). A study of the frequency of self-mutilation in a community sample of adolescents. Journal of Youth and Adolescence, 31, 67-77.

Rzeznik, J. (1998). Iris [Recorded by the Goo Goo Dolls]. On Dizzy Up The Girl [CD]. Burbank, CA: Warner Brothers Records.

Scherr, M.A., \& Walker, B. (2003). Right Now. [Recorded by Korn]. On Take a Look in the Mirror [CD]. New York, NY: Epic Records.

Solomon, Y., \& Farrano, J. (1996). "Why don't you do it properly?" Young women who selfinjure. Journal of Adolescence, 19, 111-119.

Spitzer, R.L., Kroenke, K., \& Williams, J.B.W. (1999). Validation and utility of a self-report version of Prime-MD: A PHQ primary care study. Journal of the American Medical Association, 282, 1787-1788. 
Strong, M. (1998). A bright red scream: Self-mutilation and the language of pain. New York: Penguin Group.

Whitlock, J., Eckenrode, J., \& Silverman, D. (2006). Self-injurious behaviors in a college population. Pediatrics, 117, 1939-1948.

Zanarini, M.C., Vujanovic, A.A., Parachini, E.A., Boulanger, J.L., Frankenburg, F.R., \& Hennen, J. (2003). A screening measure for BPD: The McLean screening instrument for borderline personality disorder. Journal of Personality Disorders, 17, 568-573. 